\title{
Long-Term Clustering, Scaling, and Universality in the Temporal Occurrence of Earthquakes
}

\author{
Álvaro Corral*
}

(October 25, 2018)

\begin{abstract}
Analyzing diverse seismic catalogs, we have determined that the probability densities of the earthquake recurrence times for different spatial areas and magnitude ranges can be described by a unique universal distribution if the time is rescaled with the mean rate of occurrence. The shape of the distribution shows the existence of clustering beyond the short-term regime, and scaling reveals the self-similarity of the clustering structure. This holds from worldwide to local scales and for quite different tectonic environments. Aftershock sequences also follow this universal recurrence-time distribution if the rescaling is undertaken with the time-varying rate.
\end{abstract}

Typeset using REVTEX

*Departament de Física, Facultat de Ciències, Universitat Autònoma de Barcelona, E-08193 Bellaterra, Barcelona, Spain 
Although earthquakes are a phenomenon of great complexity, certain simple general laws govern the statistics of their occurrence (1-5); however, no such a unified description has yet been established for the time interval between successive events (6-9). On the contrary, the rich variability intrinsic to earthquakes has promoted that all possibilities have been proposed, from totally random occurrence to the periodic ticking of great quakes. The most extended view is that of two separated processes, one for mainshocks, which ought to follow a Poisson distribution (10) (or not (6-9)), and an independent process to generate aftershocks. Consequently, the "standard practice" for this approach consists first of delimiting the spatial area to be studied, on the basis of its tectonic characteristics, and then of a careful (and not so standard) identification of aftershocks, in order to separate them from the main sequence.

Here we take an alternative perspective, complementary to the previous reductionist view. We try to look at the system as a whole, irrespective of tectonic features and placing all the events on the same footing, whether these would be classified as mainshocks or aftershocks (11,12). This follows one of the key guidelines of complexity philosophy, which is to find descriptions on a general level; the existence of general laws fulfilled by all the earthquakes will unveil a degree of unity in an extremely complex phenomenon (13).

We analyze a global catalog, the PDE from the NEIC (14), as well as several local catalogs: that of the SCSN (Southern California) (15), the JUNEC (Japan) (16), the Bulletins of the IGN (the Iberian Peninsula and the North of Africa) (17), and the BGS catalog (the British Islands and the North Sea) (18). Catalogs generally characterize each earthquake by three main quantities: time of occurrence, magnitude, and a vector of spatial coordinates for the hypocenter. These are also the variables that we focus on.

Without concerning ourselves with the tectonic properties, following Bak et al. (11,12), we consider spatial areas delimited by a window of $L$ degrees in longitude and $L$ degrees in latitude (this corresponds to a square region if these angles are translated onto a rectangular coordinate system (19)). For each one of these regions, only events with magnitude $M$ above a certain threshold $M_{c}$ are considered (the threshold should be larger than the minimum magnitude for which the catalog is considered complete). In this way, we transform a 
time process in four dimensions (spatial coordinates and magnitude) into a simple process on a line for which events occur at times $t_{i}$, with $i=1,2 \ldots N$, and therefore, the time between successive events can be obtained as $\tau_{i}=t_{i+1}-t_{i}, i=1,2 \ldots N-1$. These are the recurrence times in a given $L^{2}$-region for events above $M_{c}$, which can also be referred to as inter-occurrence or waiting times. Note that, with this transformation, we have lost the structure in space and in the magnitude scale; nonetheless, the change in the process properties with the variation of $L$ and $M_{c}$ will allow us to recover some of this information.

Due to the multiple time scales involved (from seconds or minutes to many years), the probability density of the recurrence time must be calculated with care. We could work with the logarithm of $\tau$, but an equivalent and more direct possibility is to define the bins over which the probability density is calculated exponentially growing as $c^{n}$, with $c>1$ and $n$ labeling consecutive bins (we usually take $c=2.5$ ). This ensures that we have the appropriate bin size for each time scale. We then count the number of pairs of consecutive events separated by a time whose value lies into a given bin, and divide by the total number of pair of events (number of events minus one) and by the size of the bin, to attain the estimation of the probability density $D_{x y}(\tau)$ over that bin, where $x y$ denotes the spatial coordinates of the region. ( $D_{x y}$ also depends on $L$ and $M_{c}$, but for the sake of simplicity in the notation, we obviate this dependence.) Moreover, due to the incompleteness of the catalogs in the short-time scale, we will not display in the plots time intervals that are smaller than 2 minutes.

The entire Earth has been analyzed by this method. Figure 1A shows the results for $D_{x y}(\tau)$ for worldwide earthquakes in the NEIC-PDE catalog for the 1973-2002 period, using $L$ from $180^{\circ}$ to $2.8^{\circ}$ (about $300 \mathrm{~km}$ ) and $M_{c}$ from 5 to 6.5 . Note the variation of the recurrence time across several orders of magnitude. Figure 1B shows the rescaling of all the distributions with the mean rate $R$ in the region, defined as the total number of events divided by the total time interval over which these events span. The perfect data collapse implies that we can write

$$
D_{x y}(\tau)=R_{x y} f\left(R_{x y} \tau\right)
$$


where $R_{x y}$ stresses that the rate refers to the $(x, y)$-region (of size $L^{2}$ and with $M \geq M_{c}$ ). The scaling function $f$ can be well fit by a generalized gamma distribution,

$$
f(\theta)=C \frac{1}{\theta^{1-\gamma}} \exp \left(-\theta^{\delta} / B\right),
$$

with parameters $\gamma=0.67 \pm 0.05, \delta=0.98 \pm 0.05, B=1.58 \pm 0.15$, and $C=0.50 \pm 0.10$, which has a coefficient of variation $C V \simeq 1.2$. In fact, the value of delta can be approximated to one, which corresponds to the standard gamma distribution; we therefore essentially have a power law with exponent about -0.33 , up to the largest values, where the exponential factor comes into play.

The scaling function fits the rescaled distributions surprisingly well for intermediate and large values of the recurrence time, about $\tau>0.01 / R_{x y}$, (this usually contains from $90 \%$ to $95 \%$ of probability). The deviations are considerable for small values of $\tau$; although the statistic is low in this case (few events in the small bins being considered), for certain regions there is a clear tendency for the distribution to exceed the value given by the scaling function, that is, there is an excess of very short recurrence times, in the form of another power law but with the exponent close to (minus) one. This occurs when the rate in the region is not stationary, due to the sudden increase and slow decay of the activity provoked by aftershock sequences. Furthermore, these increments become more apparent when the size of the region decreases, in such a way that, for $L \leq 11^{\circ}$, not all the regions in the world verify the scaling law; this can be solved in some cases by rescaling with the mean rate in the region calculated, not over the whole time-span of the catalog, but only over the period for which the rate is stationary (no activity peaks). Nevertheless, the aftershocks can be so important for certain particular regions that a stationary period may not exist.

The same analysis is performed on local catalogs and identical results hold, as Fig. 1C illustrates. For Southern California for the $1984-2001$ period, a number of small regions with stationary activity are shown; for larger regions, the time window must be reduced to $1988-1991$ or to $1995-1998$, for instance, in order to find stationariness. For Japan, we also analyze large regions for the 1995 - 1998 period, for the Iberian Peninsula the period 
is 1993 - 1997, and for the British Islands, 1991 - 2001. The magnitude thresholds range from 2 to 4 , and $L$ from $30^{\circ}$ to $0.16^{\circ}$ (approximately 3300 to $17 \mathrm{~km}$ ).

The shape of the distribution $D_{x y}(\tau)$ indicates that the memory of the last earthquake is conserved up to the largest times, with the probability of a subsequent event being maximum immediately after the last shock, and slowly decreasing with time. This constitutes a clustering effect (20), in which earthquakes attract each other, and has as a counter-intuitive consequence the fact that the longer it has been since the last earthquake, the longer the expected time will be till the next (21). On the other hand, the scaling of $D_{x y}(\tau)$ under changes in $M_{c}, L$, and the region coordinates implies that the clustering structure is selfsimilar over different regions and magnitude ranges. The robustness of the distribution under such changes is therefore noteworthy. It is also remarkable that if the region is kept fixed and only $M_{c}$ varies, the scaling with the rate $R_{x y}$ can be substituted by the factor $10^{-b M_{c}}$, where $b$ refers to the $b$-value of the frequency-magnitude relation $(1,2)$ in that particular region (11,12); despite the regional variability of $b$, the universality of the scaling function $f$ remains valid.

At the outset of this exposition, we suggested that our results were valid for all events, including aftershocks; however, the aftershocks can break the scaling of the distribution up to very large time values. We can then say that the universal distribution does not describe the short-time intervals over which aftershocks replicate. This may appear to be correct, but can be turn out to be mistaken in the following way: for aftershock sequences, the mean rate is not stationary, but changes with time; therefore, we should rescale the recurrence times using the "instantaneous" rate $r_{x y}(t)$. For many sequences, $r_{x y}(t)$ is found to decay following the Omori law (3),

$$
r_{x y}(t)=\frac{A}{t^{p}}
$$

where $t$ is the time elapsed since the mainshock (and the parameters depend on $x, y, L$, and $M_{c}$ ). Figure 2A shows precisely this for several important earthquakes in Southern California (15). The corresponding recurrence-time distributions, calculated over the period 
for which the Omori law is fulfilled, are displayed in Fig. 2B; the results after rescaling with $r_{x y}(t)$ appear in Fig. 2C, again in agreement with the universal distribution. The scaling is outstanding, taking into account that the $p$-values spread from 0.9 to 1.35. Note also that this scaling implies the existence of a secondary clustering inside the primary clustering of the aftershock sequence, and therefore the process is not a nonhomogeneous Poisson process.

The present characterization of the stochastic spatio-temporal occurrence of earthquakes by means of a unique law would indicate the existence of universal mechanisms in the earthquake-generation process (22), the understanding of which, however, is still far beyond us. Nevertheless, the context of self-organized critical phenomena (11-13) provides a coherent framework at this stage. These findings can also be relevant to continuous-time random-walk models of seismicity (23,24), time-dependent hazard, and forecasting in general (25-28).

\section{References and Notes}

1. B. Gutenberg, C. F. Richter, Seismicity of the Earth (Hafner Pub. Co., New York, $1965)$

2. D. L. Turcotte, Fractals and Chaos in Geology and Geophysics (Cambridge University Press, Cambridge, ed. 2, 1997).

3. T. Utsu, Y. Ogata, R. S. Matsu'ura, J. Phys. Earth 43, 1 (1995).

4. P. A. Reasenberg, L. M. Jones, Science 243, 1173 (1989).

5. Y. Y. Kagan, L. Knopoff, Geophys. J. R. astr. Soc. 62, 303 (1980).

6. D. Sornette, L. Knopoff, Bull. Seism. Soc. Am. 87, 789 (1997).

7. J.-H. Wang, C.-H. Kuo, J. Seism. 2, 351 (1998). 
8. W. L. Ellsworth et al., "A physically-based earthquake recurrence model for estimation of long-term earthquake probabilities" (U.S. Geological Survey Open-File Report 99$522,1999)$.

9. R. F. Smalley, J.-L. Chatelain, D. L. Turcotte, R. Prévot, Bull. Seism. Soc. Am. 77, 1368 (1987).

10. J. K. Gardner, L. Knopoff, Bull. Seism. Soc. Am. 64, 1363 (1974).

11. P. Bak, K. Christensen, L. Danon, T. Scanlon, Phys. Rev. Lett. 88, 178501 (2002).

12. K. Christensen, L. Danon, T. Scanlon, P. Bak, Proc. Natl. Acad. Sci. USA 99, 2509 (2002).

13. P. Bak, How Nature Works: The Science of Self-Organized Criticality (Copernicus, New York, 1996).

14. National Earthquake Information Center, Preliminary Determination of Epicenters catalog, http://wwwneic.cr.usgs.gov/neis/epic/epic_global.html.

15. Southern California Seismographic Network, http://www.scecdc.scec.org/ftp/catalogs/SCSN.

16. Japan University Network Earthquake Catalog, http://wwweic.eri.u-tokyo.ac.jp/CATALOG/junec/monthly.html.

17. Instituto Geográfico Nacional, Boletín de Sismos Próximos, http://www.geo.ign.es/servidor/sismo/cnis/terremotos.html.

18. British Geological Survey, catalog available upon request.

19. Nevertheless, the shape of the region is totally irrelevant in our study.

20. Y. Y. Kagan, D. D. Jackson, Geophys. J. Int. 104, 117 (1991).

21. P. M. Davis, D. D. Jackson, Y. Y. Kagan, Bull. Seism. Soc. Am. 79, 1439 (1989). 
22. S. Toda, R. S. Stein, T. Sagiya, Nature 419, 58 (2002).

23. Y. Ogata, Pure appl. geophys. 155, 471 (1999).

24. A. Helmstetter, D. Sornette, Phys. Rev. E 66, 061104 (2002).

25. I. Main, Nature 385, 19 (1997).

26. R. J. Geller, D. D. Jackson, Y. Y. Kagan, F. Mulargia, Science 275, 1616 (1997).

27. Nature Debates, "Is the reliable prediction of individual earthquakes a realistic scientific goal?" http://www.nature.com/nature/debates/index.html.

28. T. Parsons, S. Toda, R. S. Stein, A. Barka, J. H. Dieterich, Science 288, 661 (2000).

29. This work would have been impossible without the fundamental ideas put forward by the late Per Bak. The author is also grateful to M. Boguñá, K. Christensen, D. Pavón, the Ramón y Cajal program, and all the people at the Statistical Physics Group of the Universitat Autònoma de Barcelona, as well as to those institutions that have made their catalogs available on the Internet. 


\section{Figure Captions}

Fig. 1. Recurrence-time distributions without and with rescaling. (A) Probability densities from the NEIC-PDE worldwide catalog for several regions, $L$, and $M_{c}$. For $L \geq$ $45^{\circ}$, all the regions with more than 500 events are shown, whereas for $L \leq 22.5^{\circ}$, only representative regions with moderate aftershock activity are displayed. The vector $\left(k_{x}, k_{y}\right)$ labels the different regions, for which the coordinates of the center can be obtained as $x=x_{\min }+\left(k_{x}+0.5\right) L, y=y_{\min }+\left(k_{y}+0.5\right) L$, with $x_{\min }=-180^{\circ}, y_{\min }=-90^{\circ}$. (The $360^{\circ} \times 180^{\circ}$ region, which covers the whole planet, has been included for completeness.) (B) Previous data, after rescaling, with a fit of the scaling function $f$. (C) Rescaled distributions from local catalogs. SC88, SC95, SC84 refer to Southern California for the years 1988-1991, 1995 - 1998, and $1984-2001$. To obtain region coordinates, use the previous formula with $\left(x_{\text {min }}, y_{\text {min }}\right)=\left(-124^{\circ}, 29^{\circ}\right),\left(-123^{\circ}, 30^{\circ}\right),\left(125^{\circ}, 25^{\circ}\right),\left(-20^{\circ}, 30^{\circ}\right)$, and $\left(-10^{\circ}, 45^{\circ}\right)$ for SC8895, SC84, Japan, the Iberian Peninsula, and the British Islands respectively. The function displayed is the fit obtained from the NEIC-PDE catalog.

Fig. 2. Analysis of aftershock sequences. (A) Decay of the rate after a mainshock and illustration of the Omori law for the following earthquakes in Southern California: Chalfant Valley (July 21, 1986, $M=5.9$ ), Landers (June 28, 1992, $M=7.3$ ), Northridge (Jan. 17, 1994, $M=6.7$ ), and Hector Mine (Oct. 16, 1999, $M=7.1$ ). Regions of diverse size $L$ are considered, all of these including the mainshock. Some curves are shifted for the sake of clarity. (B) Distributions of recurrence times for the previous sequences. (C) Distributions $\psi$ of the dimensionless time $r_{x y}(t) \tau$, in total agreement with the universal scaling function $f$. 


\section{FIGURES}
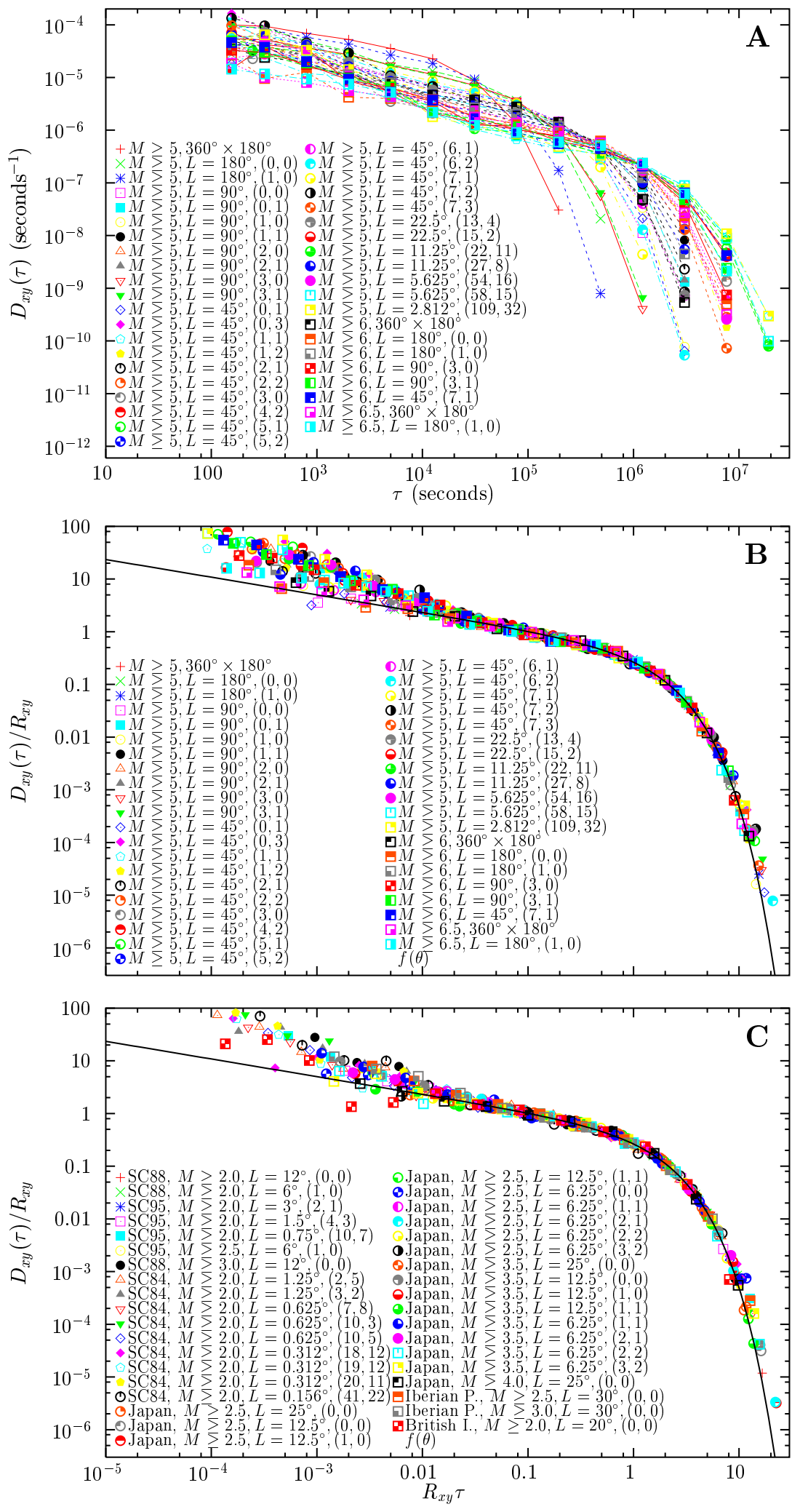

FIG. 1. 

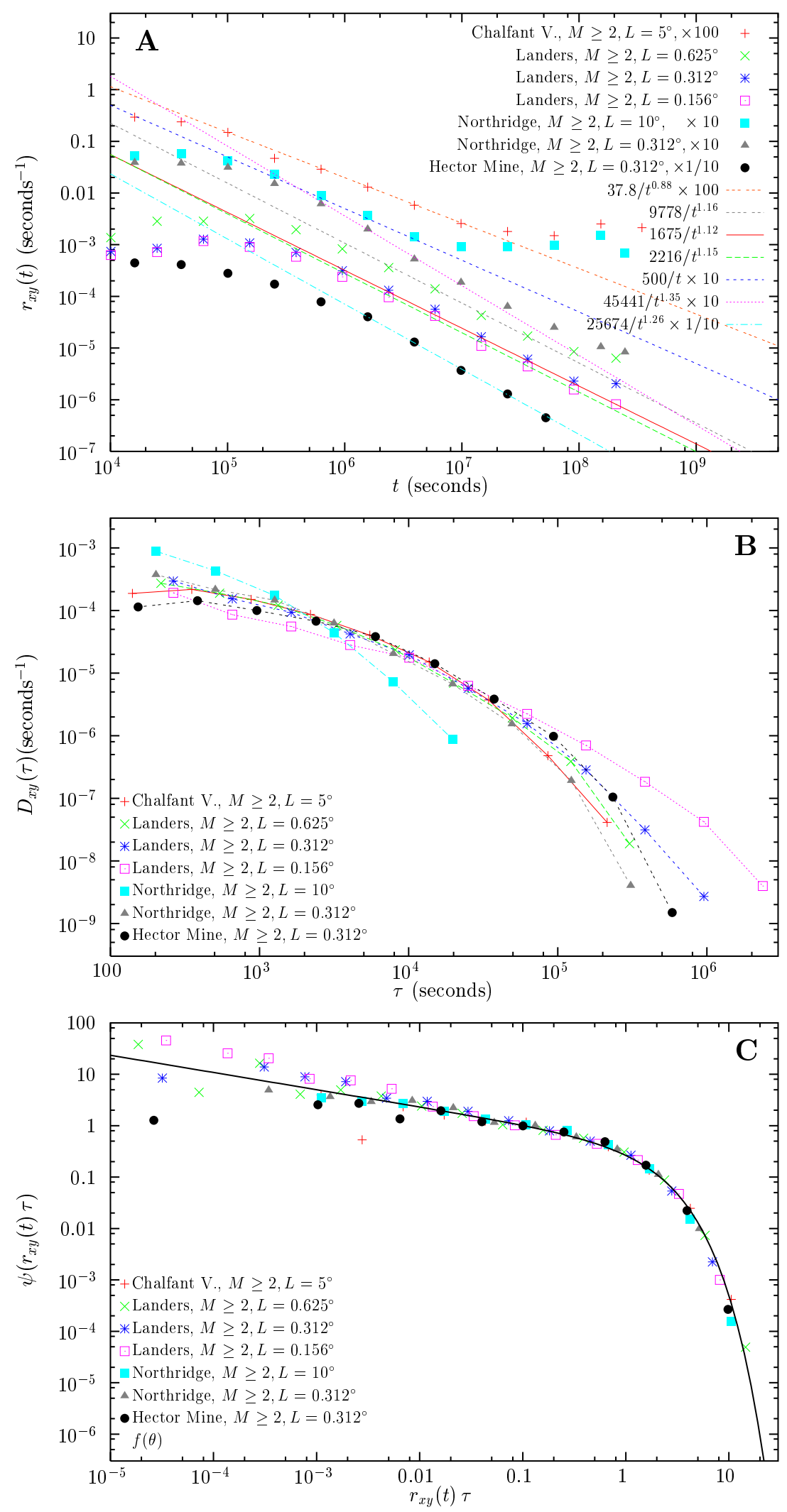

FIG. 2. 